\title{
Systems Biology and Systems Pharmacology of Thrombosis
}

\author{
M.A. Panteleev ${ }^{1,2,3,4}$ * , A.N. Sveshnikova ${ }^{1,2,3}$, A.V. Belyaev ${ }^{1,2}$, D.Y. Nechipurenko ${ }^{3}$, \\ I. Gudich ${ }^{1,2}$, S.I. Obydenny ${ }^{1,2}$, N. Dovlatova ${ }^{5}$, S.C. Fox ${ }^{5}$, E.L. Holmuhamedov 6,7 \\ ${ }^{1}$ Federal Research and Clinical Center of Pediatric Hematology \\ Oncology and Immunology, 117198 Moscow, Russia \\ 2 Center for Theoretical Problems of Physicochemical Pharmacology \\ Russian Academy of Sciences, 119991 Moscow, Russia \\ ${ }^{3}$ Faculty of Physics, Moscow State University, 119899 Moscow, Russia \\ ${ }^{4}$ National Research Center for Hematology, 125167 Moscow, Russia \\ ${ }^{5}$ Cardiovascular Medicine, University of Nottingham, Nottingham, UK \\ ${ }^{6}$ Center for Integrative Research on Cardiovascular Aging \\ Aurora University of Wisconsin Medical Group, Aurora Health Care \\ Milwaukee, Wisconsin, United States of America \\ ${ }^{7}$ Institute of Theoretical and Experimental Biophysics \\ Russian Academy of Sciences, 142290 Pushchino, Russia
}

\begin{abstract}
Thrombus formation in flowing blood is a complex time- and space-dependent process of cell adhesion and fibrin gel formation controlled by huge intricate networks of biochemical reactions. This combination of complex biochemistry, non-Newtonian hydrodynamics, and transport processes makes thrombosis difficult to understand. That is why numerous attempts to use mathematical modeling for this purpose were undertaken during the last decade. In particular, recent years witnessed something of a transition from the "systems biology" to the "systems pharmacology/systems medicine" stage: computational modeling is being increasingly applied to practical problems such as drug development, investigation of particular events underlying disease, analysis of the mechanism(s) of drug's action, determining an optimal dosing protocols, etc. Here we review recent advances and challenges in our understanding of thrombus formation.
\end{abstract}

Keywords and phrases: systems biology, systems pharmacology, thrombosis, hemostasis, platelets, blood coagulation

Mathematics Subject Classification: 92C42, 92C35, 92C50, 92C05

${ }^{*}$ Corresponding author. E-mail: mapanteleev@yandex.ru 


\section{Introduction}

Hemostasis is a protective physiological mechanism that functions to stop hemorrhage upon vascular injury. The two principal components of the blood involved in hemostasis are: i) platelets, specialized anucleated cells that adhere to the damaged tissue and form a primary plug thus reducing blood loss; ii) blood coagulation, a complex biochemical reactions responsible for conversion of liquid blood plasma into a solid fibrin gel mesh which is required to completely seal the wound. Malfunctioning of the hemostatic system is the leading cause of mortality and morbidity in the modern world.

The most prominent of the hemostatic disorders is thrombosis, manifested by intravascular formation of platelet-fibrin clots that obstruct blood flow in the vessels. Life-threatening thrombus formation has been identified as a cause of multiple diseases and conditions such as atherosclerosis, trauma, stroke, infarction, cancer, sepsis, surgery and others, and exemplified by $70 \%$ of cardiac deaths worldwide due to thrombosis [1] and approximately 400000 such deaths recorded annually in the United States only [2].

Platelet aggregation and blood coagulation are extremely complex processes. Attachment of platelets and their accumulation into thrombus is regulated by mechanical interactions with erythrocytes and vessel wall, by numerous chemical agents such as thrombin, or ADP, or prostaglandins, or collagen, as well as by an enormous network of intracellular signaling. Blood coagulation is only marginally simpler, including some fifty proteins that interact with each other and with blood or vascular cells in approximately two hundred reactions in the presence of flow and diffusion. Although extensive research during the last decades identified many key players in the hemostatic system, regulation of hemostasis and thrombosis remains poorly understood. It is extremely difficult to relate a protein or a reaction in such a complex system to the functioning of the system as a whole.

Huge complexity of thrombosis makes it an attractive target for computational modeling that can aid not only in basic research, but also in solving practical issues of diagnostics, treatment, and drug design. Actually, this complexity also makes researchers doubt usefulness of such modeling [3], which is a subject of some debate [4]. However, it can be safely assumed that computational models have, in the end, the same advantages and disadvantages as experimental models: they are a simplification that can allow better understanding, but a simplification nevertheless. However, the same can be said about any experimental model like ferric chloride-induced thrombosis that has been in use for drug design and basic research for decades, and is a subject of major criticism as one full of artifacts [5]. These days, papers with mathematical models are published not only in computational biology or biophysics journals, but also in the leading medical ones [3,4,6-19]. These practical clinical applications of computational biology are of particular interest.

Several reviews on mathematical and computational modeling in the field of hemostasis and thrombosis are available. Our previous paper covers the early period of model development with regard to blood coagulation [6], and another one focuses on clinical applications specifically [14]. There are also several recent reviews and editorials on thrombosis modeling by Xu et al. [19-21], Fasano et al [22], and Diamond [8]. However, these reviews are already somewhat outdated, and initially did not aim at the development of a comprehensive picture of applied computational modeling in hemostasis and thrombosis, which is the subject of this paper.

\section{What are all these models for?}

Why do we need mathematical and computational models of biological systems? In general, mathematical and computational modeling is a way to provide quantitative and/or semi-quantitative analysis of experimental data, which helps to better understand the nature of a phenomenon under the study. However, typical computational model includes a huge pile of partial differential equations with large number of undetermined or poorly determined parameters, which brings "vulnerability" to the model and the results generated using particular model. In this regards, the major question of computational modeling is "If there is a way accurately dissect and use this pile of equations to obtain specific answer to targeted practical problems?" 


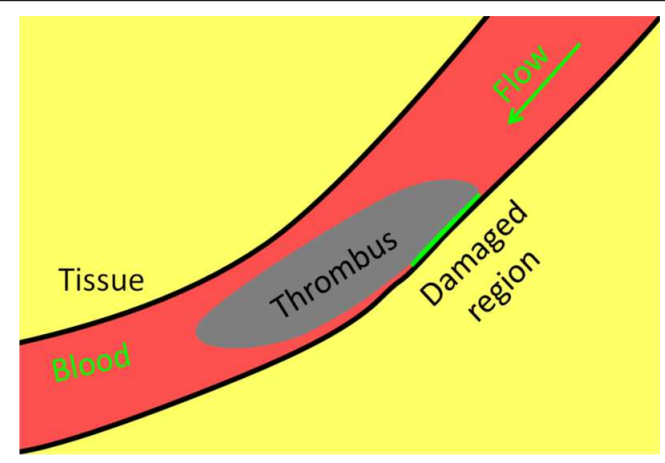

Figure 1. Thrombus formation in a vessel. The scheme shows formation of a partially occlusive thrombus in a blood vessel initiated by a contact of blood with a damaged region of the vessel wall.

The major clinical and pharmacological problems that attract the attention of systems biologists (including mathematicians) could be listed as following:

1) Investigation of the mechanisms underlying thrombus formation. It is not clear how a specific defect in the platelet-dependent hemostasis or in the coagulation system may affect the clinically observed phenotype. A typical example is the nature of bleeding in hemophilia that has been a subject of numerous investigations and hypothesis [23].

2) Identification of drugs targeting specific steps in complicated biochemical network of reactions involved in clot formation. A related problem is development of novel methods to deal with the disease. What is the optimal target for inhibition in the complicated reaction network? Is there a way to inhibit thrombosis without damaging physiological hemostasis? Various sensitivity analysis methods are the main approach to these problems [24].

3) Study of the drug mechanism of action. Even when drug is developed, the best way of its application is not always clear. Indications for treatment, correct dosing, possible drug-drug interactions etc. are usually the subject of such research, which forms a major part of systems pharmacology.

4) Personalized medicine. Finally, the models can be used not only for general problems, but also for simulation of individual cases based on the available data $[7,25,26]$.

\section{The basics of thrombosis}

Contact of blood with damaged endothelium induces the process of thrombus formation (Figure 1). Under arterial conditions, the leading mechanism of thrombus development is adhesion of platelets, that can be activated to carry out numerous functions, first of them being their ability to interact with the site of the vessel wall injury and with each other to form aggregates. Another mechanism that is more pronounced under venous flow conditions is formation of fibrin that leads to jellification of blood plasma and conversion of the protein fibrinogen into fibrin, which is controlled by enzyme thrombin, as a result of the process of blood coagulation (Figure 2).

In both cases, however, the final thrombus includes platelets as well as fibrin. A typical scanning electron microscopy image of a clot formed in whole blood is shown in Figure 3: it can be seen that red blood cells, platelets, thrombin and fibrin are all observed there.

Disturbances in any element of the hemostatic system can be a cause for bleeding or thrombosis. Deficiencies of coagulation factors, as well as insufficient quantity or functionality of platelets can be associated with various types of bleeding. In contrast, increased concentrations of many proteins such as prothrombin or fibrinogen (or deficiencies of anti-coagulation proteins), or of lipid microparticles are well-recognized risk factors for thrombosis; other risk factors include abnormalities of flow (e.g. venous thrombosis caused by prolonged flights) or vessel wall (atherosclerotic plaques). 


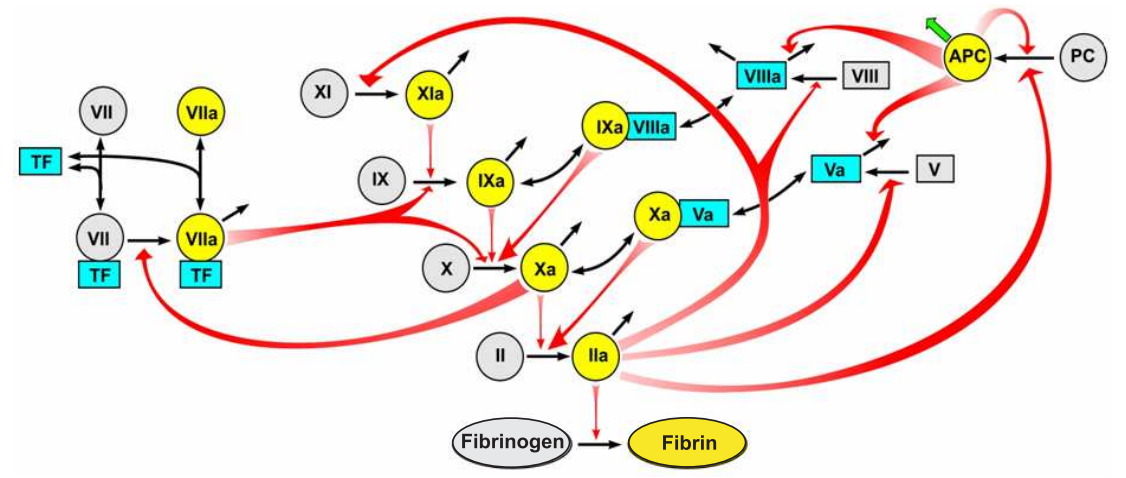

Figure 2. The blood coagulation cascade. Coagulation is a network of enzymatic reactions. The cascade enzymes are serine proteases activating each other via proteolysis. Their lifetimes are limited due to protease inhibitors. Primary activator is a transmembrane protein tissue factor (TF) which is localized to extravascular tissues and comes in contact with blood upon injury. The complex of TF and factor VIIa activates factor $\mathrm{X}$ into $\mathrm{Xa}$ that activates prothrombin into thrombin; thrombin converts fibrinogen into fibrin; fibrin polymerizes to jellificate blood. In addition, thrombin activates factors VIII, V, XI, and VII in positive feedback loops, and protein C in a negative feedback; factor VII is also activated by factor Xa. Ultimately, thrombin cleaves fibrinogen to yield fibrin that polymerizes and forms a clot. Adapted from [24].

To summarize, the main elements of the hemostatic system that are usually modeled are:

1) Platelet adhesion and aggregation in flow. The basis of arterial thrombus formation is attachment of platelets to the sites of the vessel wall injury . This requires little in terms of complicated activation mechanisms, but a rather challenging hydrodynamics problems. There are, however, additional complications such as different pro-aggregatory ability of different activated platelet subpopulations [27].

2) Platelet activation and signal transduction. Activation of each platelet that occurs at the earliest stages of the thrombus formation is an extremely complicated process that includes shape change, granule secretion, phosphatidylserine externalization etc., and is regulated by an immense signaling network that is only partially deciphered. This module does not require hydrodynamics but is extremely challenging from the biochemical point of view: most reactions are poorly known, and their parameters have not been determined.

3) Network of blood coagulation. Compared with other parts, reactions of blood coagulation are relatively well characterized. Despite numerous challenges, in particular with regard to poorly known mechanisms of the membrane-dependent reactions [28] and fibrin polymerization issues [29], this part is probably the best studied of all.

4) Fibrinolysis. Finally, dissolution of the fibrin network is a critically important process. It usually occurs at larger timescales than clot formation, and is not included into the majority of models of thrombosis.

It can be noted that the problem of thrombus formation is not only complex, it is also a multiscale one: the processes occur at vastly different spatial and temporal scales. Therefore, various multiscale approaches are an important component of most current models [21].

\section{Development of models}

Let us consider the models dealing with the main modules of the hemostatic system as described above.

1) Platelet adhesion and aggregation in flowing blood. Numerous models were developed for investigation of thrombus formation in flowing blood. First of all, the researchers almost always first analyzed 


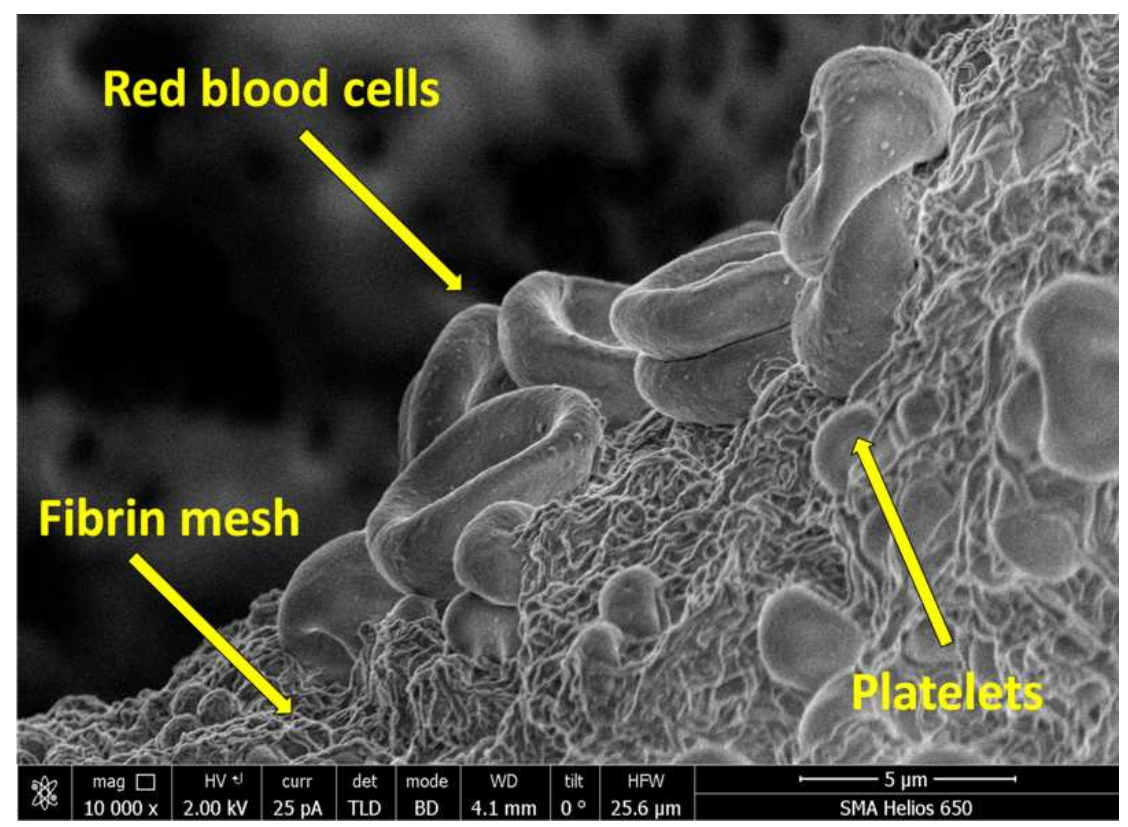

FIgURE 3. Electron microscopy imaging of the structure of the whole blood clot. Citrated whole blood was clotted by recalcification, fixed with glutaraldehyde, dried and subjected to scanning electron microscopy. Principal cellular elements of the clot (red blood cells and platelets) and fibrin network are indicated in the figure.

blood flow dynamics and platelet-flow/platelet-erythrocyte interactions that are critical for platelet adhesion. Models of blood flow with a varying degree of detalization were developed by Fogelson group [30,31], by Fedosov et al. [32-34], by the Alber group [35], by Tosenberger et al. [36], by Tokarev et al. $[37,38]$, by Mody and King [39,40], and also by others; and almost all these groups then further developed thrombus formation models. A typical example can be found in Figure 4, where three-dimensional flow of deformable erythrocytes can be observed rather realistically. The main computational models of thrombus formation developed during the last years are those by Alber group [20,41,42], by Fogelson group $[43,44]$, and others $[45,46]$. Models for some specific cases, like thrombosis is case of intracranial aneurisms, were also proposed [47].

Despite significant progress, development of these models is presently limited by computational power and methods available. Analysis of platelet-vessel wall interaction, collision with erythrocytes, and of the subpopulation phenomena requires simulation of single cells mechanics in $3 \mathrm{D}$, which is presently possible for a very little number of cells and very small volumes. Therefore, thrombus formation on the large scale usually requires simplified continuum models [48]. Another limitation is poor understanding of platelet activation mechanisms, which prevents correct description of long-term thrombus development.

2) Platelet activation and signal transduction. The first mechanism-driven model of some signaling pathways in platelets upon stimulation with adenosine dis-phosphate was created in the Diamond lab in 2008 [16], followed by a more detailed study [49]. This was recently supplemented by a model of thrombin signaling via one of thrombin receptors [50]. The cyclic adenosine monophosphate signaling has also been a subject of computational modeling [51]. However, there is no single consistent platelet signaling model, and this field is probably the least studied of all. Therefore, the majority of researchers use simplified phenomenological models when they need to include platelets into large-scale models $[27,52-54]$.

3) Network of blood coagulation. There are several mathematical models of blood coagulation that form the basis for others. One of the best characterized is the model designed by in Mann laboratory to simulate 
thrombin formation in a reconstituted system of coagulation factors and phospholipids [55]; anticoagulant reactions were added later [56]. This model formed a basis for numerous clinical applications by the same group $[4,7,13,25,26,57-60]$ and other groups [41,61]. Another model, developed by our group, differed by including spatial propagation of fibrin clot and took into account interaction of coagulation factors with platelets [52]. Since then, this model also gave rise to a number of pharmacological and basic studies $[15,24,62-65]$.

Other models for the blood coagulation network have also been developed such as [18], and some of them also were further applied for clinical research [9-12]. Some of the thrombosis models also included coagulation network in some form $[20,21,43,64,66,67]$.

Despite abundance of these models, some parts of the coagulation network are known much worse than others. Despite extensive research, there are few models that attempt to carefully simulate membranedependent reactions [68] and interaction of coagulation factors with platelet subpopulations [69,70], fibrin polymerization in flow [29], or contact pathway [71], and further development of coagulation models will likely center on these aspects.

4) Fibrinolysis. This module has received relatively little attention during the last years. The only model of thrombus growth including several fibrinolytic reactions was one of Anand et al. [66,67]. However, new detailed models of tissue plasminogen activator-initiated fibrinolysis have recently been developed $[72,73]$.

From here we shall go to the specific results obtained; all sections of this review mostly focus on recent studies, as previous advances were reviewed in [14].

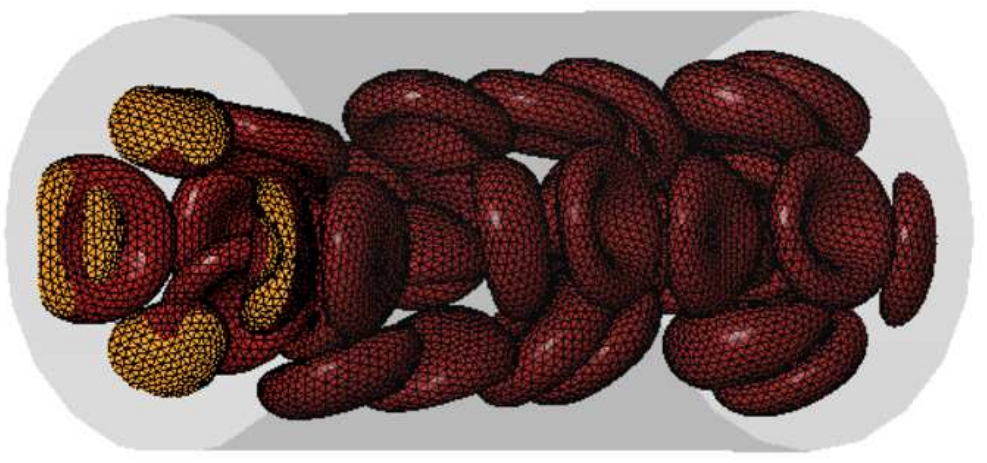

Figure 4. Red blood cells in flow: a particle dynamics simulation. The figure shows distribution of red blood cells in a three-dimensional vessel. Red blood cell shape, deformation, collisions, distribution of cells and flow velocity in the vessel are in good agreement with the experiments. Reproduced from [36].

\section{Models of thrombosis: clinical significance}

Onset of thrombus formation remains highly intriguing, in particular, with regard to the blood coagulation components. This question was analyzed using many mathematical models of blood coagulation $[24,61$, 62]. However, none of these computational models considered/included the role of blood flow in the process of clot formation (REF). Remarkably, a mathematical model of fibrin thrombus formation in the presence of flow [64] revealed that the meaning of the initiation reactions completely changes in the presence of flow-mediated transport. The positive feedback of factor VII activation by factor Xa that has no role in the absence of flow turns out to be critical for the onset of clotting in flow. These feedbacks make coagulation sensitive to flow in a threshold-like manner, abruptly stopping clot initiation once shear 
rate exceeds some threshold. This not only sheds light on the basic mechanisms of clotting initiation, but also suggests revision of the mechanisms of action of many drugs, first of all recombinant activated factor VII [74].

An even more urgent problem of the field of thrombus formation is the mystery of thrombus size regulation. Both blood coagulation and platelet thrombus growth are self-sustained process and can potentially propagate in space once initiated until complete occlusion. If this mechanism is discovered, it can be used for development of new therapeutic approaches that could differentially regulate initiation and propagation of thrombi, and could potentially regulate thrombosis/bleeding without affecting normal hemostasis. For the blood coagulation system, the protein C-dependent mechanism of clot growth termination via a negative feedback loop was demonstrated experimentally and analyzed theoretically in $[52,63]$.

For platelet thrombus formation, one possibility is hindered transport of coagulation factors: prothrombin is completely depleted in the region where prothrombinase is assembled, an no new prothrombin can get there [75]. Another hypothesis was proposed in [20]: it was suggested that a fibrin "cap" is formed on the surface of thrombus that prevents further platelet attachment; this was further explored in [76]. Although there is some experimental evidence that a fibrin cap or shell is observed in vivo and can stop thrombus growth [77], another group using a well-established experimental model of thrombosis reported that fibrin is located in the core of the thrombus rather than in "shell" or "cap" [78] and thus cannot stop thrombus growth.

Another concept, also based on the interaction of the platelet- and plasma-dependent hemostasis, was suggested by our group in [53], and further developed in a more detailed model in [54]. According to this concept, fibrin is not likely to be formed on the surface of platelet thrombi in arterial shear rates [64]. Therefore, fibrin is formed within the thrombus; however, the regions of platelet thrombus above the fibrin core are at some time point disrupted and removed by flow, which externalizes the inert fibrin shell and stops further propagation (Figure 5).

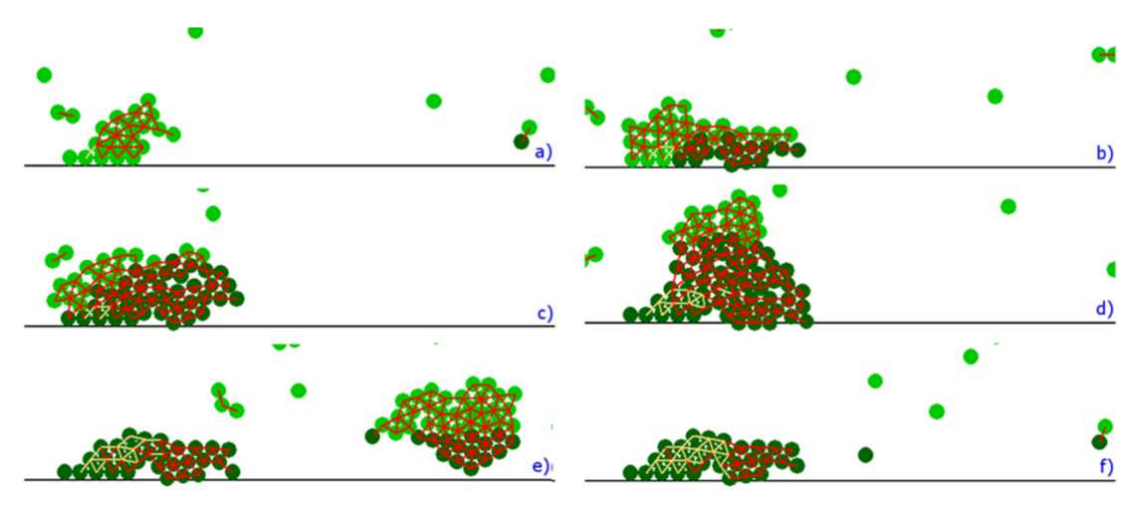

FiguRE 5. Stopping thrombus growth as a result of disruption of the shell and externalization of the fibrin core. Snapshots of the clot growth for the hybrid model: a) the clot begins to form, b) fibrin begins to form in the growing clot, c) clot core is full of fibrin but the clot continues to grow, d) the clot reaches its critical size, e) the clot ruptures and its outer part is removed by flow, f) the core of the clot remains captured in fibrin mesh, which prevents the clot from growing further. Reproduced from [54]. 
M.A. Panteleev, A.N. Sveshnikova, A.V. Belyaev, D.Y. Nechipurenko, I. Gudich, S.I. Obydenny, N. Dovlatova, S.C. Fox, E.L. Holmuhamedov Systems biology and systems pharmacology of thrombosis

\section{Blood coagulation: sensitivity analysis, modules, variability}

An important group of methods that is usually employed to better understand a complex system is sensitivity analysis: individual parameters are perturbed in order to estimate impact of these parameters on the model behavior. Sensitivity analysis has several objectives: 1) to identify reactions and processes that are important for the system functioning; 2) to see whether experimental errors in the parameter determination allow their use in the model (e.g. a huge error is not critical if the parameter is not important); 3) to study the effect of variability of the parameters within their normal value ranges.

All these applications can be found with regard to blood coagulation models. Interestingly, there are few examples of sensitivity analysis with regard to thrombus formation models [64], probably because these models represent the ultimate goal of computational hemostasis and thrombosis, and are still in their early stage of development.

Identification of the "critical elements" in the coagulation network was first carried out by Luan et al. [79], who found that thrombin and factor Xa are the best targets with regard to overall potency. Impact of uncertainty in parameter determination on the model behavior was studied by Danforth et al. [59]. Finally, boundaries of normal hemostasis and effects of individual variability were explored in [26] and [11]. A typical example can be found in Figure 6.

The most sophisticated type of sensitivity analysis was introduced in our paper [24], where we called it "task-oriented analysis". The basic idea behind this method is that biological systems usually have several tasks, and different output parameters are specific for each task. Therefore, there is not one, but several sensitivity coefficients that allow identification of specific subsets of processes responsible for the control of different tasks carried out by the system. Furthermore, perturbation was carried out by two methods, by either small perturbations or by removal of components in order to evaluate both local sensitivity and integral importance. Finally, we mapped all important components for each output to identify sub-systems, or modules, responsible for each task. Figure 7 displays the resulting modular decomposition of the coagulation network, where different modules are identified that are responsible for different tasks carried out by this system. This is critical for both diagnostic and drug development purposes because this allows to segregate clotting response into several aspects that can be differentially regulated. Further application of this method can be found in [64].

\section{Other clinical applications: systems pharmacology and beyond}

Investigation of the molecular mechanisms underlying disease(s). Among disorders of hemostasis, major attention with regard to the modeling was attracted by hemophilia, deficiency of coagulation factors VIII, IX, or XI. The nature of this disease is not clear because there are alternative pathway of coagulation that do not involve these factors and should allow sufficient thrombin formation. We found that these factors are important components of positive feedback loops that control spatial propagation of coagulation $[52,63]$, which can have important consequences for diagnostics and treatment $[23,62,65]$.

Two other cases that were subject of investigation by Mitrofanov et al. are coagulopathies caused by decreased temperature [12] and hemodilution $[10,11]$. Both these disorders are common and their understanding is very complicated because both factors (temperature and dilution) affect both pro- and anti-coagulant reactions, so that the resulting shift of balance is not intuitively obvious. Finally, one more interesting example is the study by Butenas et al. on the significance of circulating factor IXa in blood that can cause thrombosis [58].

Study of the mechanism(s) of drug's action. One of the two main lines of computational research with regard to the already established drugs is investigation of new anticoagulants. All these studies were carried out using the model developed in the Mann group. The model was used to differentiate effects of direct factor $\mathrm{Xa}$ inhibitor and low molecular weight heparin on acute and ongoing coagulation processes [13]. Investigation of the anticoagulant mechanism of action depending on the original plasma composition was continued in [60]. 


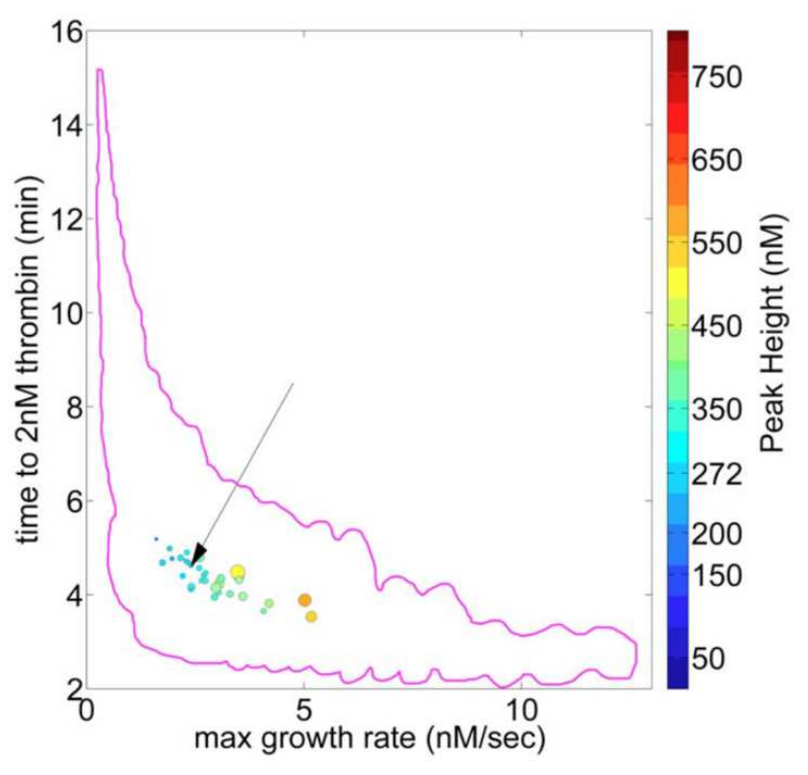

FIGURE 6. Computationally derived thrombin generation phenotypes in a population of apparently healthy individuals. Plasma factor composition for 32 individuals was used to generate time courses of thrombin generation, thrombin parameters were extracted and each individual. The boundaries (magenta) of the theoretical population are outlined and an individual with all factors at their mean physiologic values is also presented. Arrow indicates an individual with all factors at mean physiological. Reproduced from [26].

Another line that received significant attention is pharmacology of bypassing agents in hemophilia. These drugs are used to compensate factor VIII or IX deficiency via alternative mechanisms (that do not include replacement of the missing factors), which can be important if hemophilia is associated with development of antibodies against these factors rendering replacement therapy inefficient. One such agent is recombinant activated factor VII, which has been in use for decades, but whose mechanisms of action are still a matter of debate [74]. Computational modeling was used to obtain novel information with regard to this enzyme: a critical role of flow in the regulation of this molecule was identified [64]; differential effects of rVIIa on thrombin generation was studied [9]; and ultimately, a model unifying its mechanism of action depending on the conditions was proposed [17].

An interesting alternative to rVIIa is inactivation of plasma inhibitors, e.g. of tissue factor pathway inhibitor; in other words, inhibition of inhibitors means stimulation. Our group investigated one such candidate drug, an inhibitor aptamer BAX-499 in the spatial model of clot growth and demonstrated how this molecule can improve coagulation in hemophilia depending on factor VIII activity and tissue factor density [15]. Computational modeling was a critical tool for experimental planning and data analysis in that study.

Personalized medicine. Finally, mathematical modeling can be used for diagnostics and dose adjustment in individual patients, because the models can identify parameters of the clotting system that are not readily detected in experiments. A classic example is use of modeling to study factor Xa generation based on the determined coagulation factors levels [57]; the same group used computational approaches for the analysis of prothrombotic phenotype [25] and of other phenotypes [7]. In all cases, coagulation factor levels were determined and integrated into the thrombin generation model to evaluate overall procoagulant potential and to identify the reasons behind the thrombotic risk. 
A similar approach for the platelet-dependent part of hemostasis was recently employed to analyze thrombus formation in patients [80]. Patient platelet response was determined using large-throughput scanning, immersed into a multiscale model of platelet deposition, and compared with microfluidic experiments on thrombus growth. This allowed not only prediction of phenotype, but also identification of novel mutations and drug responses.

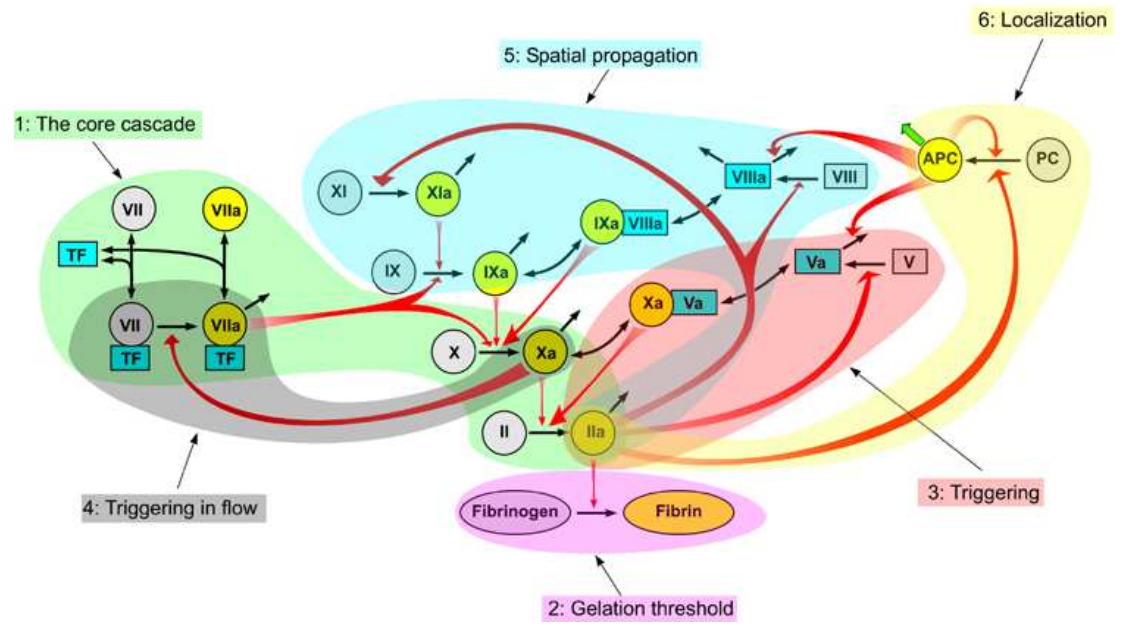

Figure 7. Modular decomposition of the coagulation network. Task-oriented modular analysis allows identification of specific modules responsible for individual functions of coagulation: threshold behavior, spatial propagation, etc. Reproduced from [24].

\section{Conclusion}

Thrombosis is a critically important field of medicine, where computational models are not only a great tool for research, but are also in high demand by both medical community and pharmacological companies. Despite numerous challenges in both biological and mathematical parts of this field, the next decade will likely witness mathematical models becoming a major integral part of decision-making in diagnostics, treatment, and drug development.

Acknowledgements. We thank Prof. Vitaly Volpert (University of Lyon) and Aleksey Mazurov (Russian Cardiology Research and Production Center) for careful reading of the manuscript, critical comments and valuable suggestions. The authors are supported by the Russian Foundation for Basic Research grants 13-04-00401 and 1404-00670, and by the Russian Academy of Sciences Presidium Basic Research Programs "Molecular and Cellular Biology" and "Basic Science for Medicine".

\section{References}

[1] M.J. Davies. The pathophysiology of acute coronary syndromes. Heart, 83 (2000), 361-366.

[2] State-specific mortality from sudden cardiac death -United States, 1999. MMWR Morb. Mortal. Wkly. Rep., 51 (2002), $123-126$

[3] H.C. Hemker, S. Kerdelo, R.M. Kremers. Is there value in kinetic modeling of thrombin generation? No (unless...). J. Thromb. Haemost., 10 (2012), 1470-1477.

[4] K.G. Mann. Is there value in kinetic modeling of thrombin generation? Yes. J. Thromb.Haemost., 10 (2012), 14631469.

[5] J.D. Barr, A.K. Chauhan, G.V. Schaeffer, J.K. Hansen, D.G. Motto. Red blood cells mediate the onset of thrombosis in the ferric chloride murine model. Blood, 121 (2013), 3733-3741. 
M.A. Panteleev, A.N. Sveshnikova, A.V. Belyaev, D.Y. Nechipurenko, I. Gudich, S.I. Obydenny, N. Dovlatova, S.C. Fox, E.L. Holmuhamedov Systems biology and systems pharmacology of thrombosis

[6] F.I. Ataullakhanov, M.A. Panteleev. Mathematical modeling and computer simulation in blood coagulation. Pathophysiol. Haemost. Thromb., 34 (2005), 60-70.

[7] K.E. Brummel-Ziedins, T. Orfeo, F.R. Rosendaal, A. Undas, G.E. Rivard, S. Butenas, K.G. Mann. Empirical and theoretical phenotypic discrimination. J. Thromb. Haemost., 7 Suppl 1 (2009), 181-186.

[8] S.L. Diamond. Systems biology to predict blood function. Journal of Thrombosis and Haemostasis, 7 (2009), $177-180$.

[9] A.Y. Mitrophanov, J. Reifman. Kinetic modeling sheds light on the mode of action of recombinant factor VIIa on thrombin generation. Thromb. Res., 128 (2011), 381-390.

[10] A.Y. Mitrophanov, F.R. Rosendaal, J. Reifman. Therapeutic correction of thrombin generation in dilution-induced coagulopathy: computational analysis based on a data set of healthy subjects. J. Trauma Acute. Care Surg., 73 (2012), S95-S102.

[11] A.Y. Mitrophanov, F.R. Rosendaal, J. Reifman. Computational analysis of intersubject variability and thrombin generation in dilutional coagulopathy. Transfusion, 52 (2012), 2475-2486.

[12] A.Y. Mitrophanov, F.R. Rosendaal, J. Reifman. Computational Analysis of the Effects of Reduced Temperature on Thrombin Generation: The Contributions of Hypothermia to Coagulopathy. Anesth. Analg., 2013.

[13] T. Orfeo, S. Butenas, K.E. Brummel-Ziedins, M. Gissel, K.G. Mann. Anticoagulation by factor Xa inhibitors. J. Thromb. Haemost., 8 (2010), 1745-1753.

[14] M.A. Panteleev, N.M. Ananyeva, F.I. Ataullakhanov, E.L. Saenko. Mathematical models of blood coagulation and platelet adhesion: clinical applications. Curr. Pharm. Des, 13 (2007), 1457-1467.

[15] L.A. Parunov, O.A. Fadeeva, A.N. Balandina, N.P. Soshitova, K.G. Kopylov, M.A. Kumskova, J.C. Gilbert, R.G. Schaub, K.E. McGinness, F.I. Ataullakhanov, M.A. Panteleev. Improvement of spatial fibrin formation by the antiTFPI aptamer BAX499: changing clot size by targeting extrinsic pathway initiation. J. Thromb. Haemost., 9 (2011), $1825-1834$.

[16] J.E. Purvis, M.S. Chatterjee, L.F. Brass, S.L. Diamond. A molecular signaling model of platelet phosphoinositide and calcium regulation during homeostasis and P2Y1 activation. Blood, 112 (2008), 4069-4079.

[17] A.M. Shibeko, S.A. Woodle, T.K. Lee, M.V. Ovanesov. Unifying the mechanism of recombinant FVIIa action: dose dependence is regulated differently by tissue factor and phospholipids. Blood, 120 (2012), 891-899.

[18] T. Wajima, G.K. Isbister, S.B. Duffull. A comprehensive model for the humoral coagulation network in humans. Clin. Pharmacol. Ther., 86 (2009), 290-298.

[19] Z. Xu, M. Kamocka, M. Alber, E.D. Rosen, Computational approaches to studying thrombus development. Arterioscler. Thromb. Vasc. Biol., 31 (2011), 500-505.

[20] Z. Xu, S. Christley, J. Lioi, O. Kim, C. Harvey, W. Sun, E.D. Rosen, M. Alber. Multiscale model of fibrin accumulation on the blood clot surface and platelet dynamics. Methods Cell Biol., 110 (2012), 367-388.

[21] Z. Xu, O. Kim, M. Kamocka, E.D. Rosen, M. Alber. Multiscale models of thrombogenesis. Wiley. Interdiscip. Rev. Syst. Biol. Med., 4 (2012), 237-246.

[22] A. Fasano, R.F. Santos, A. Sequeira. Blood coagulation: A puzzle for biologists, a maze for mathematicians. Modeling of Physiological Flows, Springer, (2012), 41-75.

[23] F.I. Ataullakhanov, N.M. Dashkevich, C. Negrier, M.A. Panteleev. Factor XI and traveling waves: the key to understanding coagulation in hemophilia? Expert. Rev. Hematol., 6 (2013), 111-113.

[24] M.A. Panteleev, A.N. Balandina, E.N. Lipets, M.V. Ovanesov, F.I. Ataullakhanov. Task-oriented modular decomposition of biological networks: trigger mechanism in blood coagulation. Biophys. J., 98 (2010), 1751-1761.

[25] K.E. Brummel-Ziedins, T. Orfeo, P.W. Callas, M. Gissel, K.G. Mann, E.G. Bovill. The prothrombotic phenotypes in familial protein $C$ deficiency are differentiated by computational modeling of thrombin generation. PLoS.One., 7 (2012), e44378

[26] C.M. Danforth, T. Orfeo, S.J. Everse, K.G. Mann, K.E. Brummel-Ziedins. Defining the boundaries of normal thrombin generation: investigations into hemostasis. PLoS One., 7 (2012), e30385.

[27] A.O. Yakimenko, F.Y. Verholomova, Y.N. Kotova, F.I. Ataullakhanov, M.A. Panteleev. Identification of different proaggregatory abilities of activated platelet subpopulations. Biophys. J., 102 (2012), 2261-2269.

[28] M.A. Panteleev, N.M. Ananyeva, N.J. Greco, F.I. Ataullakhanov, E.L. Saenko. Factor VIIIa regulates substrate delivery to the intrinsic factor X-activating complex. FEBS J., 273 (2006), 374-387.

[29] R.D. Guy, A.L. Fogelson, J.P. Keener. Fibrin gel formation in a shear flow. Math. Med. Biol., 24 (2007), 111-130.

[30] L.M. Crowl, A.L. Fogelson. Computational model of whole blood exhibiting lateral platelet motion induced by red blood cells. Int.j.numer.method.biomed.eng, 26 (2010), 471-487.

[31] T. Skorczewski, L.C. Erickson, A.L. Fogelson. Platelet motion near a vessel wall or thrombus surface in twodimensional whole blood simulations. Biophys. J., 104 (2013), 1764-1772.

[32] D.A. Fedosov, B. Caswell, G.E. Karniadakis. A multiscale red blood cell model with accurate mechanics, rheology, and dynamics. Biophys. J., 98 (2010), 2215-2225.

[33] D.A. Fedosov, H. Lei, B. Caswell, S. Suresh, G.E. Karniadakis. Multiscale modeling of red blood cell mechanics and blood flow in malaria. PLoS Comput.Biol., 7 (2011), e1002270.

[34] D.A. Fedosov, H. Noguchi, G. Gompper. Multiscale modeling of blood flow: from single cells to blood rheology. Biomech. Model. Mechanobiol., 2013.

[35] C.R. Sweet, S. Chatterjee, Z. Xu, K. Bisordi, E.D. Rosen, M. Alber. Modelling platelet-blood flow interaction using the subcellular element Langevin method. J.R. Soc. Interface, 8 (2011), 1760-1771.

[36] A. Tosenberger, V. Salnikov, N. Bessonov, E. Babushkina, V. Volpert. Particle dynamics methods of blood flow simulations. Mathematical Modelling of Natural Phenomena, 6 (2011), 320-332. 
M.A. Panteleev, A.N. Sveshnikova, A.V. Belyaev, D.Y. Nechipurenko, I. Gudich, S.I. Obydenny, N. Dovlatova, S.C. Fox, E.L. Holmuhamedov Systems biology and systems pharmacology of thrombosis

[37] A.A. Tokarev, A.A. Butylin, E.A. Ermakova, E.E. Shnol, G.P. Panasenko, F.I. Ataullakhanov. Finite platelet size could be responsible for platelet margination effect. Biophys. J., 101 (2011), 1835-1843.

[38] A.A. Tokarev, A.A. Butylin, F.I. Ataullakhanov. Platelet adhesion from shear blood flow is controlled by near-wall rebounding collisions with erythrocytes. Biophys. J., 100 (2011), 799-808.

[39] N.A. Mody, M.R. King. Influence of Brownian motion on blood platelet flow behavior and adhesive dynamics near a planar wall. Langmuir, 23 (2007), 6321-6328.

[40] W. Wang, N.A. Mody, M.R. King. Multiscale model of platelet translocation and collision. J. Comput. Phys., 244 (2013), 223-235.

[41] Z. Xu, N. Chen, M.M. Kamocka, E.D. Rosen, M. Alber. A multiscale model of thrombus development. J.R.Soc.Interface, 5 (2008), 705-722.

[42] Z. Xu, J. Lioi, J. Mu, M.M. Kamocka, X. Liu, D.Z. Chen, E.D. Rosen, M. Alber. A multiscale model of venous thrombus formation with surface-mediated control of blood coagulation cascade. Biophys. J., 98 (2010), 1723-1732.

[43] A.L. Fogelson, Y.H. Hussain, K. Leiderman, Blood clot formation under flow: the importance of factor XI depends strongly on platelet count. Biophysical journal, 102 (2012), 10-18.

[44] K. Leiderman, A.L. Fogelson. Grow with the flow: a spatial-temporal model of platelet deposition and blood coagulation under flow. Mathematical Medicine and Biology, 28 (2011), 47-84.

[45] N. Filipovic, M. Kojic, A. Tsuda. Modelling thrombosis using dissipative particle dynamics method. Philosophical Transactions of the Royal Society A: Mathematical, Physical and Engineering Sciences, 366 (2008), $3265-3279$.

[46] H. Kamada, Y. Imai, M. Nakamura, T. Ishikawa, T. Yamaguchi. Computational study on thrombus formation regulated by platelet glycoprotein and blood flow shear. Microvasc. Res., 89 (2013), 95-106.

[47] S. Zimny, B. Chopard, S. Malaspinas, E. Lorenz, K. Jain, S. Roller, J. Bernsdorf. A multiscale approach for the coupled simulation of blood flow and thrombus formation in intracranial aneurysms. Procedia Computer Science, 18 (2013), $1006-1015$.

[48] A.L. Fogelson, R.D. Guy. Platelet-wall interactions in continuum models of platelet thrombosis: formulation and numerical solution. Math. Med. Biol., 21 (2004), 293-334.

[49] J.E. Purvis, R. Radhakrishnan, S.L. Diamond. Steady-state kinetic modeling constrains cellular resting states and dynamic behavior. PLoS computational biology, 5 (2009), e1000298.

[50] L. Lenoci, M. Duvernay, S. Satchell, E. DiBenedetto, H.E. Hamm. Mathematical model of PAR1-mediated activation of human platelets. Mol. Biosyst., 7 (2011), 1129-1137.

[51] G. Wangorsch, E. Butt, R. Mark, K. Hubertus, J. Geiger, T. Dandekar, M. Dittrich. Time-resolved in silico modeling of fine-tuned cAMP signaling in platelets: feedback loops, titrated phosphorylations and pharmacological modulation. BMC. Syst. Biol., 5 (2011), 178.

[52] M.A. Panteleev, M.V. Ovanesov, D.A. Kireev, A.M. Shibeko, E.I. Sinauridze, N.M. Ananyeva, A.A. Butylin, E.L. Saenko, F.I. Ataullakhanov. Spatial propagation and localization of blood coagulation are regulated by intrinsic and protein C pathways, respectively. Biophys. J., 90 (2006), 1489-1500.

[53] A. Tosenberger, F. Ataullakhanov, N. Bessonov, M. Panteleev, A. Tokarev, V. Volpert. Modelling of thrombus growth and growth stop in flow by the method of dissipative particle dynamics. Russian Journal of Numerical Analysis and Mathematical Modelling, 27 (2013), 507-522.

[54] A. Tosenberger, F. Ataullakhanov, N. Bessonov, M. Panteleev, A. Tokarev, V. Volpert. Modelling of thrombus growth in flow with a DPD-PDE method. J. Theor. Biol., 337 (2013), 30-41.

[55] K.C. Jones, K.G. Mann. A model for the tissue factor pathway to thrombin. II. A mathematical simulation. J. Biol. Chem., 269 (1994), 23367-23373.

[56] M.F. Hockin, K.C. Jones, S.J. Everse, K.G. Mann. A model for the stoichiometric regulation of blood coagulation. J. Biol. Chem., 277 (2002), 18322-18333.

[57] K.E. Brummel-Ziedins, T. Orfeo, M. Gissel, K.G. Mann, F.R. Rosendaal. Factor Xa generation by computational modeling: an additional discriminator to thrombin generation evaluation. PLoS.One., 7 (2012), e29178.

[58] S. Butenas, T. Orfeo, M.T. Gissel, K.E. Brummel, K.G. Mann. The significance of circulating factor IXa in blood. J. Biol. Chem., 279 (2004), 22875-22882.

[59] C.M. Danforth, T. Orfeo, K.G. Mann, K.E. Brummel-Ziedins, S.J. Everse. The impact of uncertainty in a blood coagulation model. Math. Med. Biol., 26 (2009), 323-336.

[60] T. Orfeo, M. Gissel, S. Butenas, A. Undas, K.E. Brummel-Ziedins, K.G. Mann. Anticoagulants and the propagation phase of thrombin generation. PLoS. One., 6 (2011), e27852.

[61] M.S. Chatterjee, W.S. Denney, H. Jing, S.L. Diamond. Systems biology of coagulation initiation: kinetics of thrombin generation in resting and activated human blood. PLoS computational biology, 6 (2010), e1000950.

[62] A.N. Balandina, A.M. Shibeko, D.A. Kireev, A.A. Novikova, I.I. Shmirev, M.A. Panteleev, F.I. Ataullakhanov. Positive feedback loops for factor $V$ and factor VII activation supply sensitivity to local surface tissue factor density during blood coagulation. Biophys. J., 101 (2011), 1816-1824.

[63] N.M. Dashkevich, M.V. Ovanesov, A.N. Balandina, S.S. Karamzin, P.I. Shestakov, N.P. Soshitova, A.A. Tokarev, M.A. Panteleev, F.I. Ataullakhanov. Thrombin activity propagates in space during blood coagulation as an excitation wave. Biophys. J., 103 (2012), 2233-2240.

[64] A.M. Shibeko, E.S. Lobanova, M.A. Panteleev, F.I. Ataullakhanov. Blood flow controls coagulation onset via the positive feedback of factor VII activation by factor Xa. BMC. Syst. Biol., 4 (2010), 5.

[65] A.A. Tokarev, Y.V. Krasotkina, M.V. Ovanesov, M.A. Panteleev, M.A. Azhigirova, V.A. Volpert, F.I. Ataullakhanov, A.A. Butilin. Spatial Dynamics of Contact-Activated Fibrin Clot Formation in vitro and in silico in Haemophilia B: Effects of Severity and Ahemphil B Treatment. Mathematical Modelling of Natural Phenomena, 1 (2006), $124-137$. 
M.A. Panteleev, A.N. Sveshnikova, A.V. Belyaev, D.Y. Nechipurenko, I. Gudich, S.I. Obydenny, N. Dovlatova, S.C. Fox,

[66] M. Anand, K. Rajagopal, K.R. Rajagopal. A model incorporating some of the mechanical and biochemical factors underlying clot formation and dissolution in flowing blood: review article. Journal of Theoretical Medicine, 5 (2003), $183-218$.

[67] M. Anand, K. Rajagopal, K.R. Rajagopal. A model for the formation, growth, and lysis of clots in quiescent plasma. A comparison between the effects of antithrombin III deficiency and protein $C$ deficiency. Journal of theoretical biology, 253 (2008), 725-738.

[68] M.A. Panteleev, E.L. Saenko, N.M. Ananyeva, F.I. Ataullakhanov. Kinetics of Factor X activation by the membranebound complex of Factor IXa and Factor VIIIa. Biochem. J., 381 (2004), 779-794.

[69] Y.N. Kotova, F.I. Ataullakhanov, M.A. Panteleev. Formation of coated platelets is regulated by the dense granule secretion of adenosine 5'diphosphate acting via the P2Y12 receptor. J. Thromb. Haemost., 6 (2008), $1603-1605$.

[70] M.A. Panteleev, N.M. Ananyeva, N.J. Greco, F.I. Ataullakhanov, E.L. Saenko. Two subpopulations of thrombinactivated platelets differ in their binding of the components of the intrinsic factor X-activating complex. J. Thromb. Haemost., 3 (2005), 2545-2553.

[71] A.V. Pokhilko, F.I. Ataullakhanov. Contact activation of blood coagulation: trigger properties and hysteresis. Kinetic recognition of foreign surfaces upon contact activation of blood coagulation: a hypothesis. J. Theor. Biol., 191 (1998), $213-219$.

[72] B.E. Bannish, J.P. Keener, M. Woodbury, J.W. Weisel, A.L. Fogelson. Modelling fibrinolysis: 1D continuum models. Math. Med. Biol., 2012.

[73] B.E. Bannish, J.P. Keener, A.L. Fogelson. Modelling fibrinolysis: a 3D stochastic multiscale model. Math. Med. Biol., 2012.

[74] M.V. Ovanesov, M.A. Panteleev, E.I. Sinauridze, D.A. Kireev, O.P. Plyushch, K.G. Kopylov, E.G. Lopatina, E.L. Saenko, F.I. Ataullakhanov. Mechanisms of action of recombinant activated factor VII in the context of tissue factor concentration and distribution. Blood Coagul. Fibrinolysis, 19 (2008), 743-755.

[75] K. Leiderman, A.L. Fogelson. The Influence of Hindered Transport on the Development of Platelet Thrombi Under Flow. Bull. Math. Biol., 2012.

[76] O.V. Kim, Z. Xu, E.D. Rosen, M.S. Alber. Fibrin Networks Regulate Protein Transport during Thrombus Development. PLoS Comput. Biol., 9 (2013), e1003095.

[77] M.M. Kamocka, J. Mu, X. Liu, N. Chen, A. Zollman, B. Sturonas-Brown, K. Dunn, Z. Xu, D.Z. Chen, M.S. Alber, E.D. Rosen. Two-photon intravital imaging of thrombus development. J. Biomed. Opt., 15 (2010), 016020.

[78] T.J. Stalker, E.A. Traxler, J. Wu, K.M. Wannemacher, S.L. Cermignano, R. Voronov, S.L. Diamond, L.F. Brass. Hierarchical organization in the hemostatic response and its relationship to the platelet-signaling network. Blood, 121 (2013), 1875-1885.

[79] D. Luan, M. Zai, J.D. Varner. Computationally derived points of fragility of a human cascade are consistent with current therapeutic strategies. PLoS computational biology, 3 (2007), e142.

[80] M.H. Flamm, T.V. Colace, M.S. Chatterjee, H. Jing, S. Zhou, D. Jaeger, L.F. Brass, T. Sinno, S.L. Diamond. Multiscale prediction of patient-specific platelet function under flow. Blood, 120 (2012), 190-198. 